\title{
Playing with Social and Emotional Game Companions
}

\author{
Andry Chowanda ${ }^{1,2 \star}$, Martin Flintham ${ }^{1}$, Peter Blanchfield ${ }^{1}$, Michel Valstar $^{1}$ \\ 1 School of Computer Science, The University of Nottingham, Nottingham, UK-GB \\ \{psxac6,pszmdf,pszpxb,pszmv\}@nottingham.ac.uk \\ 2 School of Computer Science, Bina Nusantara University, Jakarta, ID
}

\begin{abstract}
This paper presents the findings of an empirical study that explores player game experience by implementing the ERiSA Framework in games. A study with Action Role-Playing Game (RPG) was designed to evaluate player interactions with game companions, who were imbued with social and emotional skill by the ERiSA Framework. Players had to complete a quest in the Skyrim game, in which players had to use social and emotional skills to obtain a sword. The results clearly show that game companions who are capable of perceiving and exhibit emotions, are perceived to have personality and can forge relationships with the players, enhancing the player experience during the game.
\end{abstract}

Keywords: Game Experience, Game Companions, Social Relationship, Social Interaction

\section{Introduction}

Games are considered to be one of the most popular interactive entertainment products in the world. The key point that makes them popular is the experience people have when they play the game [13]. Non-Player Characters (NPCs) in games can be a key factor to engage a player and bring about this experience. They can be particularly interesting potential vehicles of affect, because players naturally engage with the NPCs as part of a game. Over time, a pattern of interactions between a player and agents may translate into a relationship, if the game and its NPCs are designed to accommodate this. We argue that NPCs with such capabilities will provide a new experience when playing games.

This paper presents the evaluation of player experience when playing with game companions that are capable of perceiving and exhibiting emotions, complete with the ability to develop simple social relations over time. A game scenario where players interact with two different game companions was designed by adopting the ERiSA Framework [8][10]. The ERiSA Framework is an integrated framework for social and emotional game companions to enhance their believability and quality of interaction, in particular by allowing a game companion to forge social relations and make appropriate use of social signals.

\footnotetext{
* Corresponding Author
} 
Our experimental results provide clear evidence that there is an increase in player experience when playing the game with the additional influence of interactive emotion, personality and relationships to the game companion's behaviours. Players reported that they were more emotionally involved and attached with the characters and the game when the Framework was activated in the game.

\section{Related Work}

A key goal in developing game companions one can relate to is that they should be believable. The term "believable" can be linked to the era of the 1990's when Joseph Bates argued the notion of a "believable character" in his paper [4]. He elaborated that the idea of "believability" doesn't necessarily come from a reliable character but "one that provides the illusion of life, and thus permits the audience's suspension of disbelief" [4]. The term "illusion of life" itself was coined by the Disney animators in the 1930's in their quest to make audiences believe in their characters [4][15]. The ultimate goal in this research is therefore not as much creating NPCs that are more and more realistic, but ones that less frequently break the illusion. In games, implementing the illusion results in an increase of player experience. Charles [7] argues that player experience and enjoyment can be significantly enhanced when the game character is well designed. Afonso \& Prada [1] also discuss that "Social Believability" in games improved the experience. Bailey et al. [3] suggest that the character's believability influences the player immersion and thus enhances the game-play experience.

Attfield et al. [2] define the user experience as "the emotional, cognitive and behavioural connection that exists [...] between a user and a resource". That connection can be linked to emotion, personality and social relationship aspects. The influence of those aspects on an NPC's behaviour has been widely recognised to affect their believability [4][15]. The uniqueness of a character in comparison to others in the game and their ability to exhibit emotions provides a feeling of immersion to the player. We can also see the ability of the player to forge relationships with NPCs in games as part of their storyline such as Skyrim, Grand Theft Auto, The Sims, Harvest Moon, etc. Research has been done to enhance the player experience by improving their believability. However, only limited research has been done to incorporate emotion, personality and social relationships models together (e.g. [14], [9], [10]). This paper demonstrates a comprehensive evaluation of the player experience when playing with social and emotional game companions with stereotypical artificial personalities.

\section{Designing Social \& Emotional Game Companions}

The ERiSA Framework was implemented inside the popular commercial game Skyrim. Our framework allows Skyrim to capture a player's facial expressions as an additional game input. Fig. 1 illustrates the integration of the components and the communication between them. Partial parts from the ERiSA Framework were used to perceive and interpret the player's emotions, conveyed by their 


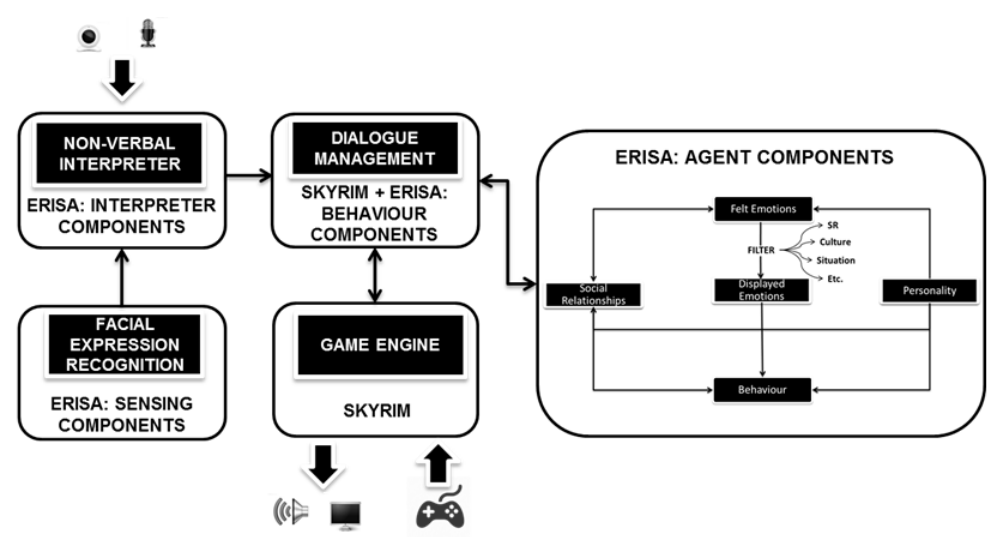

Fig. 1. Framework Implementation

facial expression and recorded by camera. The dialogue manager proposes NPC actions and determines a set of possible dialogues for the player to choose from, depending on the player's and NPC's internal states recorded in the Interpreter and Agent Components. The output corresponding to the selected NPC action is sent to the Skyrim Game Engine. A player's facial expressions also govern the player's dialogue options. For example, the option for the player to say that they're happy is only available if the facial expression recognition components interpret the player's emotion conveyed by their facial expression as happy [10].

We designed two characters with opposite personality to interact with, named Stella and Max Erisa. Stella has a high Extraversion trait, while Max has a strong Neuroticism trait. To associate the characters with their personality traits, we set Max's voice characteristic to an agitated and annoying voice provided by The Skyrim Creation Kit. On the other hand, Stella's voice is pleasantly happy and enthusiastic. Moreover, we designed a simple quest named "The Erisa Family". In the quest, the player can build relationships with the NPCs, get information about The Legendary Swords possessed by their family and receive one of the swords. Players can dynamically interact with the NPCs, with different options at their disposal depending on their relationship level. The quest is finished when the player gets one of the swords. The Sword of Friendship will be given to the player when they earned the NPCs' trust by building a positive relationship, while The Sword of Hatred will be passed to the player when their relationship is going towards a negative direction. There are 4 strategies for forging social relationship with the NPCs: chatting, giving favourite items vs. giving undesirable items, praising vs. criticising, and exploring Skyrim world together vs. attacking the NPCs. Stella is more sociable compared to Max, hence, extra effort is required to gain Max's trust. Over time, the topics discussed with the NPCs change depending on the level of their relationship with the player. Fig.2 illustrates the mapping between possible interactions corresponding to the level of relationship based on the models proposed in [10]. 


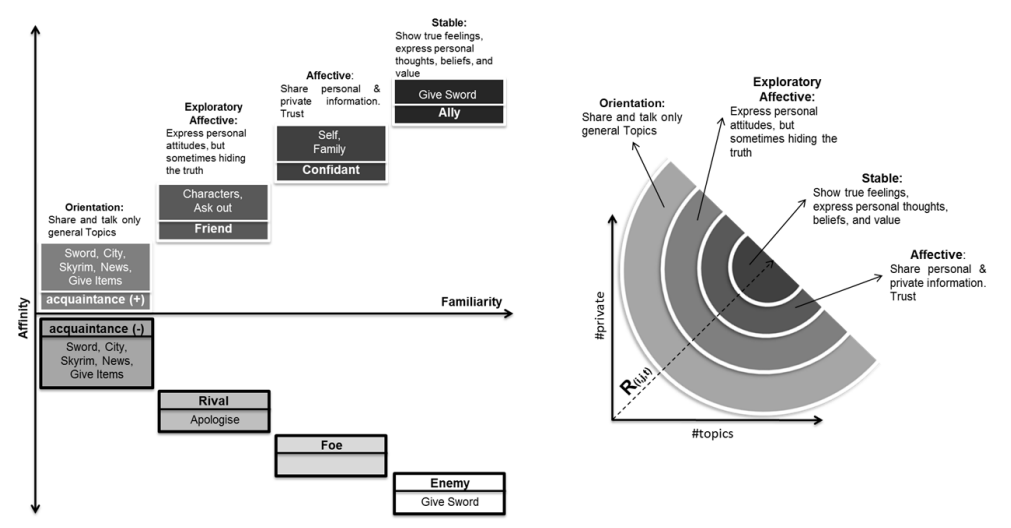

Fig. 2. Mapping Social Relationship to Behaviour

There are total of 8 relationship levels ( 4 positive, 4 negative). On the initial interaction, the player can only discuss general topics about the swords, city, Skyrim, give items, or ask and provide news. Assuming the relationship is going towards positive direction, the NPCs start to express their personal attitudes towards the topics in the next stage. Moderate topics are also available to be discussed in this stage in addition to the previous topics. Starting from this stage, the player can suggest to the NPCs to go out and explore Skyrim together. Going towards an affective relationship, the NPCs begin to share their personal information about themselves and their family. Moreover, the NPCs strongly express their personal attitudes towards the topics at this stage. In the final stage, the NPCs begin to show their true feelings, express personal thoughts, beliefs and values. At this stage the player is given The Sword of Friendship, marking the end of the quest. The player can still interact with the NPCs but the relationship between them will not go any further. On the other hand, if the relationship is changing in negative direction, the breadth and depth of topics does not change. Eventually, the Sword of Hatred is passed to the player, marking the end of the journey. If this route is taken, the player will not be able to interact with the NPCs any more, reflecting the deep hatred that the NPCs feel towards the player.

To evaluate the characters created and empowered by the ERiSA Framework, we compare them with their baseline versions. The baseline characters were designed with the original mechanics provided by Skyrim, where the NPC's emotions and social relationships are triggered by story or click-based system. The dialogues between player and the baseline NPC were only regulated by the Skyrim Dialogue Manager where the emotions conveyed by the player's facial expressions were not included in the decision making. Other than that, both the conversation topics and the quest design were identical for both baseline and models NPCs. 


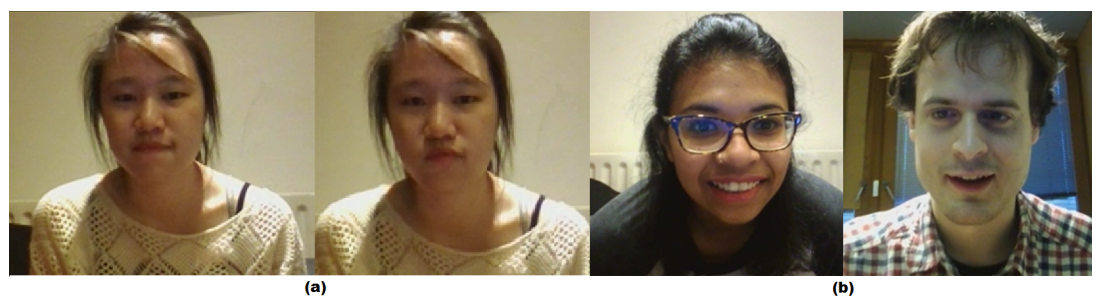

Fig. 3. Player's Facial Expressions: Annoyed by Max (a), Delighted by Stella (b)

\section{Evaluating Social \& Emotional Game Companions}

Fifteen players (60\% female, $73.33 \%$ Asian, $26.67 \%$ Caucasian, AVG age $=24.6$ ) were recruited to play the game. All were familiar with the RPG Genre and one third had played Skyrim before. A total of 60 interactions were completed with both of the NPCs, totalling 18.16 hours of play time (MAX $=51.82 \mathrm{mins}$, $\mathrm{MIN}=1.85$ mins, AVG $=18.16$ mins $)$. Each participant interacted with both NPCs without the models as a baseline and with the models implemented as the comparison. To avoid order bias, the order was randomized for all players. All interactions were video and audio recorded, the player and NPCs' internal states were logged and the player's in-game choices were recorded. Players were asked to complete a questionnaire every time they finished a session of the game. Nine players were invited to do a short interview to discuss their experience with the game, after they had finished all the quests.

The questionnaires consists of four parts, each in the form of a five-point Likert Scale evaluating the game and its NPCs. The first part evaluates the NPCs' personality as perceived by the user based on the Big Five Personality Inventory [12]. The second part rates the social relationship between the player and the NPCs based on Quality of Relationships Inventory(QRI) and Social Relationship Index(SRI) [6]. In the third part, players were asked about their feelings when they interacted with the NPCs during the quest using Ekman's six basic emotions. Finally, the last part of the questionnaire evaluated player experience during the game using The Game Engagement Questionnaire [5] and The Immersive Experience Questionnaire [11].

Fig.4 describes Max and Stella's personality as perceived by players for both models with * mark represent significance at the 0.05 level and others marked with ** indicates significance at the 0.01 level. Without the models implemented, the NPCs' appear to be perceived as devoid of any personality by the players. With the models implemented, the characteristics which constitute Stella personality, are evidently perceived by players as a person who has positive thinking, generates a lot of enthusiasm, is outspoken, outgoing \& sociable, is relaxed \& handles stress well, is emotionally stable \& not easily upset and is polite to others. Max, on the other hand, is a person who is extremely negative thinking, does not generate a lot of enthusiasm, tends to be slightly quiet, is reserved, sometimes depressed, blue \& can't handle stress well, can be tense \& worries a 


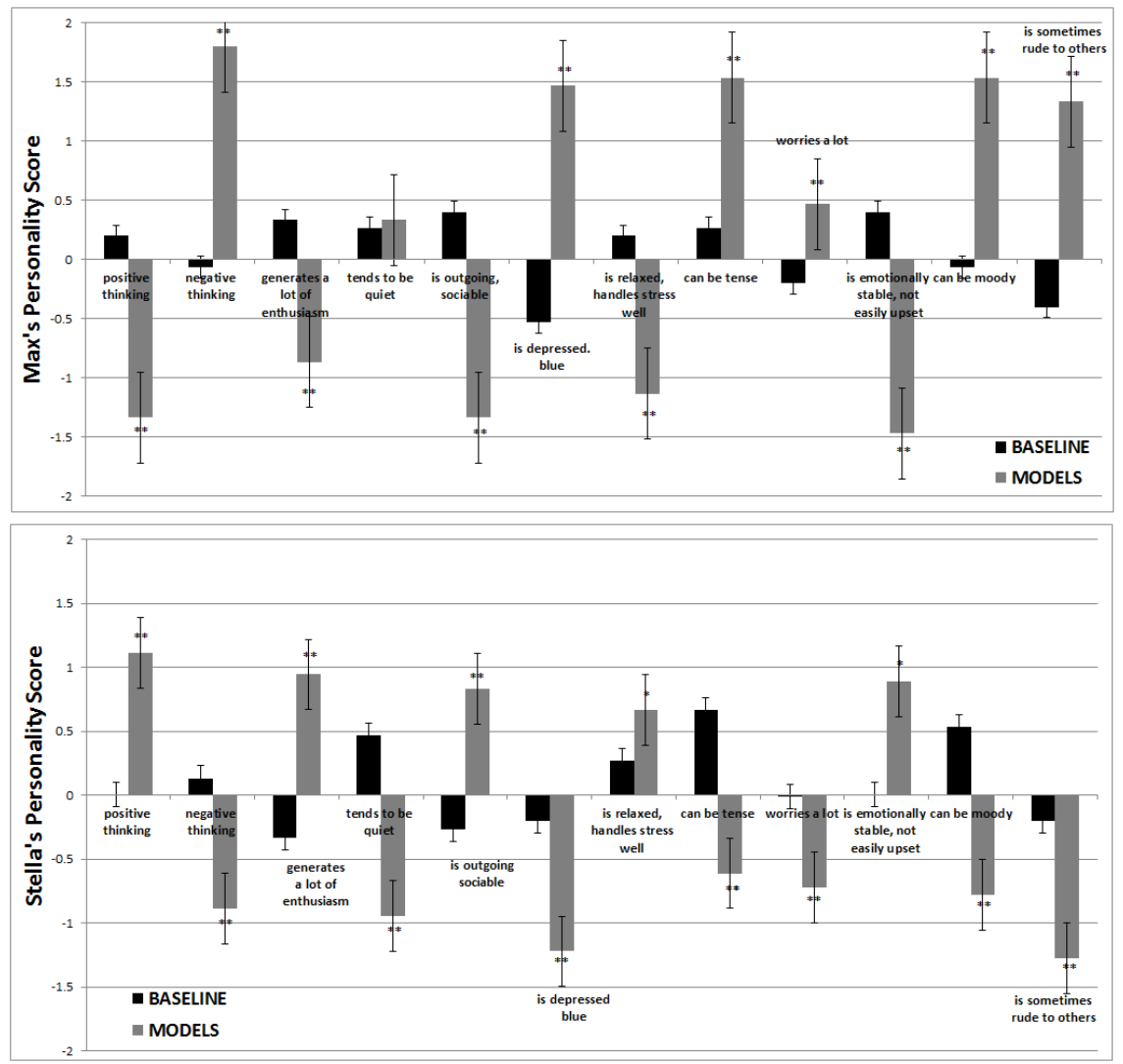

Fig. 4. Max's (top) and Stella's (bottom) Personality as Perceived by Players

lot, is easily upset, can be moody and is sometimes rude to others. In addition, most players identified Max as rude, abominable and playing hard-to-get, while Stella is a talkative, nice and approachable person. According to responses to the Game Engagement and Immersive Experience Questionnaire (see Fig. 6), players empathise with Stella but not with Max in the game when the models are implemented, while there was no significant difference when models were not implemented. Fig. 3 shows some player facial expressions when interacting with the ERISA-empowered characters.

Furthermore, players reported significant changes in their social relationship to both NPCs (see Fig. 5). With the models implemented, Stella is more likeable, and players perceived her as someone that they can count on to listen when they are very angry with someone else. Players seems happy when they interacted with her. In contrast, Max is more unpleasant to interact with, and players feel he is someone that they can't count on to listen when they are very angry with someone else as they already feel emotionally upset towards Max. Players also feel more familiar and close to both NPCs when the models are implemented. 


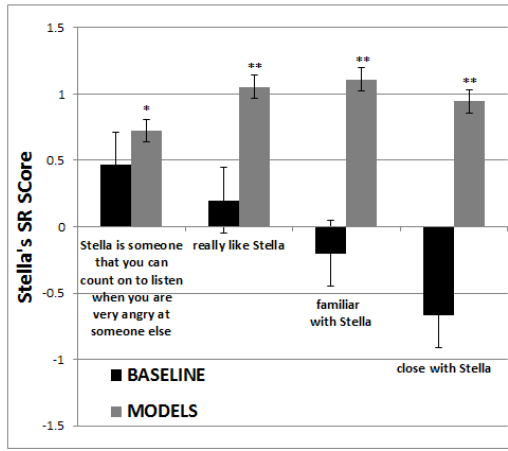

(a)

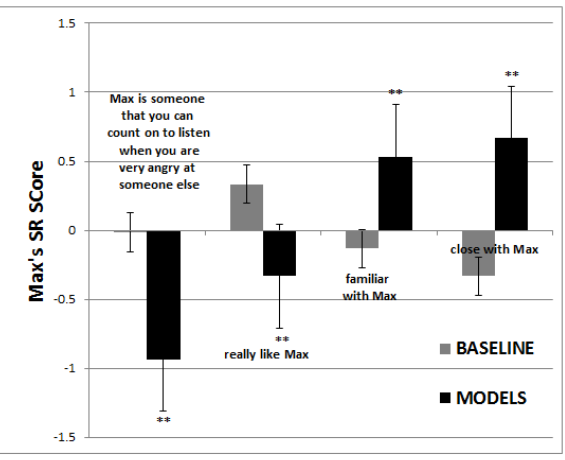

(b)

Fig. 5. Stella's (a) and Max's (b) Social Relationship as Rated by Players

Measured in in-game-time, players spent on average 0.92 hours (MAX $=$ 3.2 hours, $\mathrm{MIN}=0.25$ hours $) / 62.78$ hours $(\mathrm{MAX}=114.2$ hours, $\mathrm{MIN}=$ 11.75 hours) with Stella without and with models, respectively. Players spent 1.01 hours $(\mathrm{MAX}=4.0$ hours, $\mathrm{MIN}=0.21$ hours $) / 74.74$ hours $(\mathrm{MAX}=$ 219.5 hours, MIN = 16.17 hours) for Max with no models and with models implemented, respectively. Players who took a longer time to finish the quest spent their time mostly exploring the Skyrim world with the NPCs, while players with shorter time only focussed on forging a social relationship with the NPCs by giving them their most-liked items repeatedly. The favourite strategy to build relationships with the NPCs was giving favourable items to them, with exploring Skyrim together as the second choice of strategy. Building a positive relationship with Max can be quite a nerve-racking experience; $40 \%$ of players realised that it would be easier to have a negative relationship and decided to go towards negative relation with Max although they already held a positive relationship with him as their game strategy to get a Legendary Sword.

Fig. 6 illustrates the score of the player experience. With ERISA models implemented, players feel more engaged and emotionally immersed in the game and the characters, irrespective of which NPC they interact with. Players reported a significant increase in the engagement level with the game with the models implemented $(p<0.01)$. A significant difference was also found in the players' emotional attachment to the game and the characters with the models implemented $(p<0.01)$. Moreover, the models enriched the game story, increased the players' curiosity how the game would progress $(p<0.01)$. From the short interview, most of players preferred the NPCs with models. The models provide an interesting flow to forge the relationships with NPCs. However, some players suggested adding more variation of interactions, as the current interaction is limited to speaking, giving items, and exploring Skyrim together.

Automatic arousal and valence annotation was performed from the interaction audio using the SSI Framework [16]. In addition, we also manually annotated players' arousal and valence using continuous annotation tools developed 


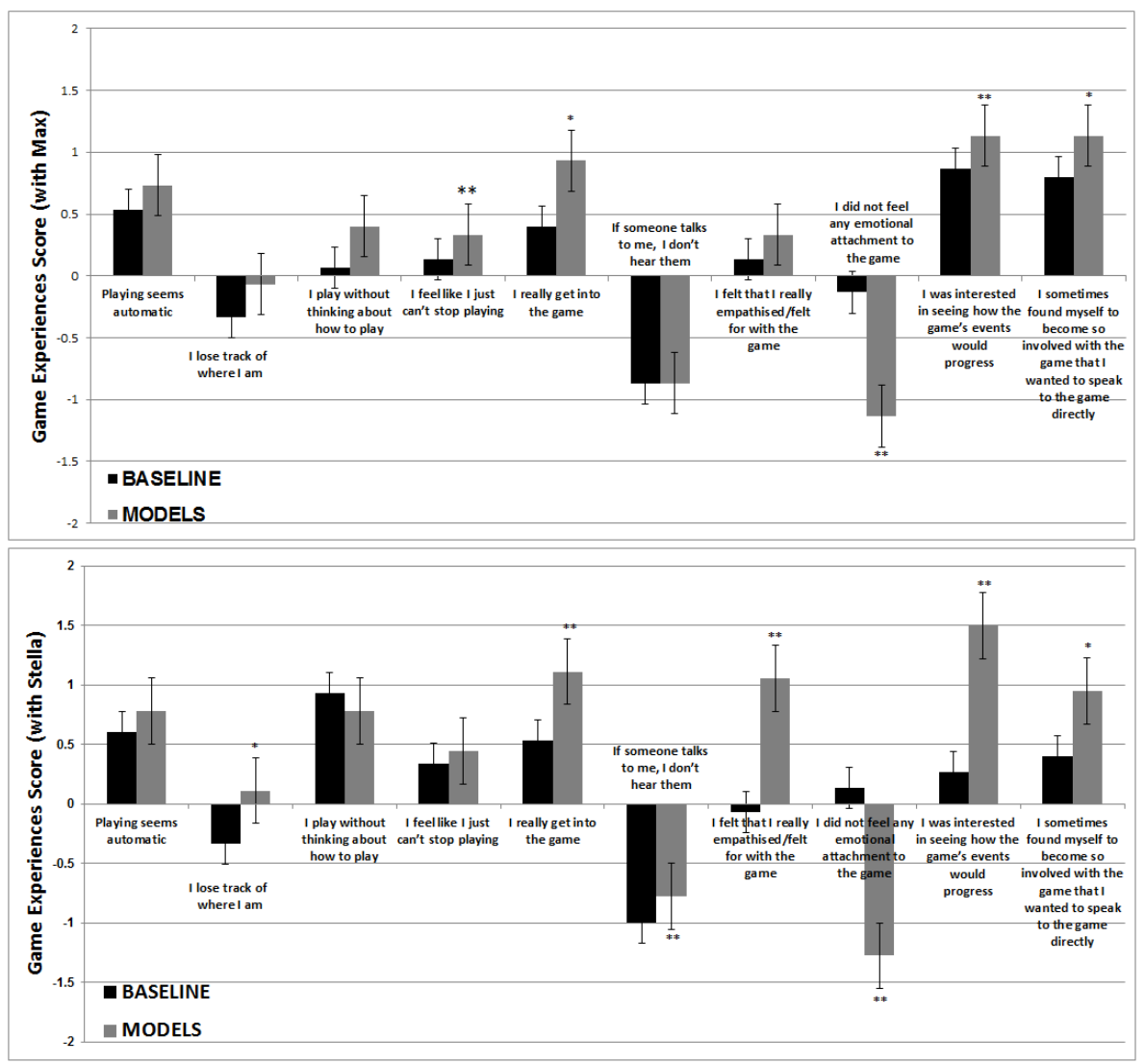

Fig. 6. Players' Experiences with Max (top) and Stella (bottom)

by The Imperial College London. A Kolmogorov-Smirnov test was applied to all the data and indicated that they were more likely come from a non-normally distributed population $(p<0.001)$. Hence Wilcoxon Signed-Rank Tests were applied as this is a good alternative to the t-Test Paired Two Sample for Means when the population cannot be assumed to be normally distributed.

Fig. 7 demonstrates the players' arousal and valence when playing with Max (left) and Stella (right). A Wilcoxon Signed-Rank Tests indicates a strong positive correlation between players' valence data with Max from automatic and manual annotation, $r=0.76, n=101, p<0.001$ and $r=0.72, n=101, p<$ 0.001 , with baseline and models implemented respectively. In addition, there was also a strong positive correlation between player' arousal data with Max from automatic and manual annotation, $r=0.62, n=101, p<0.001$ and $r=0.77, n=101, p<0.001$, with baseline and models implemented respectively. A strong positive correlation was also found in the player' valence data with Stella from automatic and manual annotation, $r=0.72, n=101, p<0.001$ 

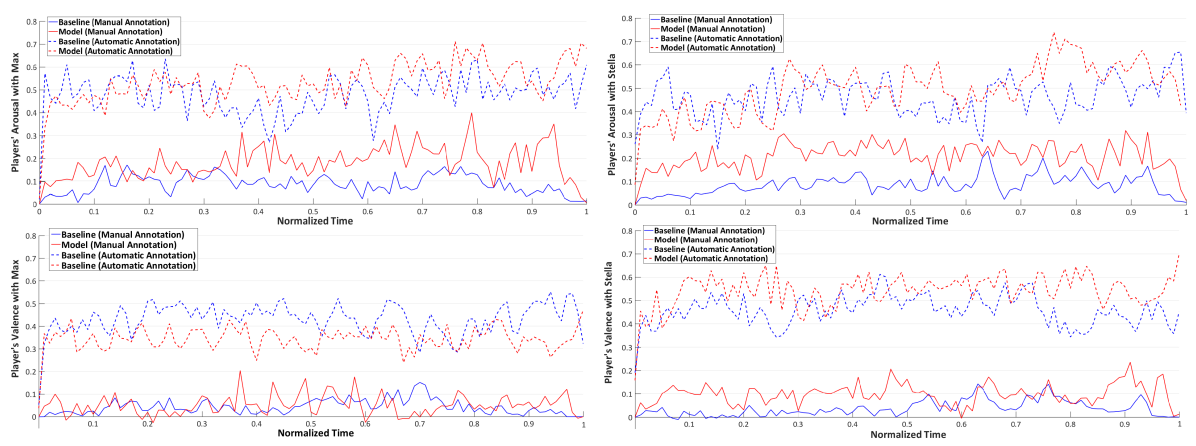

Fig. 7. Players' Arousal (top), Valence (bottom) towards Max (left), Stella (right), where dotted line represents Automatic Annotation

and $r=0.71, n=101, p<0.001$, with baseline and models implemented respectively. Moreover, there was also a strong positive correlation between player' arousal data with Stella from automatic and manual annotation, $r=0.68, n=$ $101, p<0.001$ and $r=0.83, n=101, p<0.001$, with baseline and models implemented respectively.

A Wilcoxon Signed-Ranks Test indicated that players' arousal was statically significantly higher with models implemented, $Z=8.4, p<0.001$ and $Z=$ $8.71, p<0.001$, with Max and Stella respectively. In addition, players' valence was also significantly higher with models implemented, $Z=3.1, p<0.05$ and $Z=7.75, p<0.001$, with Max and Stella respectively. Negative valence also occurred in the interaction with ERISA-empowered Max. Players' arousal and valence were significantly higher when playing with ERISA-empowered Stella compared to Max, $Z=3.75, p<0.001$ and $Z=6.47, p<0.01$, respectively. On the contrary, there was no significant differences between players' arousal and valence when interacting with baseline Stella compare to Max, $Z=-1.67, p=$ 0.5 and $Z=-1.65, p=0.1$, respectively.

\section{Conclusion and Future Work}

The results indicate that the influence of emotion, personality and social relationships to the game companions' behaviours, enhanced the player experience when playing the game. Players were emotionally involved and attached to the characters and the game. The models provide a more realistic manner for interacting with NPCs and forge relationships with them. Personality also influences the game play, as the characters with the Neuroticism trait were harder to "conquered" thus requiring a specific strategy to win. Social relationships models can be adjusted to enrich the game story as well. For future research direction with these models, more variation for interactions with the NPCs can be added. Interaction such as accomplishing a quest together and more conversational topics can enhance the user experience when playing the game. 


\section{Acknowledgement}

The work by A. Chowanda and M. Valstar is partly funded by European Union's Horizon 2020 research and innovation programme under grant agreement No 645378, ARIA-VALUSPA.

\section{References}

1. N. Afonso and R. Prada. Agents that relate:improving the social believability of non-player characters in role-playing games. In ICEC, pages 34-45. Springer, 2008.

2. S. Attfield, G. Kazai, M. Lalmas, and B. Piwowarski. Towards a science of user engagement. In WSDM workshop on user modelling for Web applications, 2011.

3. C. Bailey, J. You, G. Acton, A. Rankin, and M. Katchabaw. Believability through psychosocial behaviour: Creating bots that are more engaging and entertaining. In Believable Bots, pages 29-68. Springer, 2013.

4. J. Bates. The role of emotion in believable agents. Commun. ACM, 37(7):122-125, July 1994.

5. J. Brockmyer and et al. The development of the game engagement questionnaire: A measure of engagement in video game-playing. Journal of Experimental Social Psychology, 45(4):624-634, 2009.

6. R. Campo and et al. The assessment of positivity and negativity in social networks: The reliability and validity of the social relationships index. Journal of Community Psychology, 37(4):471-486, 2009.

7. D. Charles. Enhancing Gameplay: Challenges for Artificial Intelligence in Digital Games. In 1st World Conference on Digital Games, The Netherlands, 2003.

8. A. Chowanda, P. Blanchfield, M. Flintham, and M. Valstar. Erisa: Building emotionally realistic social game-agents companions. In T. Bickmore, S. Marsella, and C. Sidner, editors, Intelligent Virtual Agents, volume 8637 of Lecture Notes in Computer Science, pages 134-143. Springer, 2014.

9. A. Chowanda, P. Blanchfield, M. Flintham, and M. Valstar. Play smile game with erisa: A user study on game companions. In Workshop on Engagement in Social Intelligent Virtual Agents on IVA, 2015.

10. A. Chowanda, P. Blanchfield, M. Flintham, and M. Valstar. Computational models of emotion, personality, and social relationships for interactions in games. In J. Thangarajah, K. Tuyls, C. Jonker, and S. Marsella, editors, Proceedings of the 2016 International Conference on AAMAS, Singapore. 2016.

11. C. Jennett and et al. Measuring and defining the experience of immersion in games. International journal of human-computer studies, 66(9):641-661, 2008.

12. O. John, L. Naumann, and C. Soto. Paradigm shift to the integrative big five trait taxonomy. Handbook of personality: Theory and research, 3:114-158, 2008.

13. N. Lazzaro. Why we play games: Four keys to more emotion without story. 2004.

14. M. Ochs, N. Sabouret, and V. Corruble. Simulation of the dynamics of nonplayer characters' emotions and social relations in games. Computational Intelligence and AI in Games, IEEE Transactions on, 1(4):281-297, Dec 2009.

15. F. Thomas and O. Johnson. Disney Animation: The Illusion of Life. Abbeville Press, 1984.

16. J. Wagner, F. Lingenfelser, T. Baur, I. Damian, F. Kistler, and E. André. The social signal interpretation (SSI) framework: Multimodal signal processing and recognition in real-time. In Proceedings of the 21st ACM International Conference on Multimedia, pages 831-834, 2013. 